\section{TUBERCULOSIS OF THE CERVICAL LYMPHATIC GLANDS}

BY

\author{
R. REID, M.S.Lond., F.R.C.S. \\ Consulting Surgeon
}

AND

\section{C. WILKINSON, M.B.Lond.}

Medical Superintendent

Essex County Council Sanatorium, Black Notley

Tuberculosis of the lymphatic glands is a disease that tends to run a long and debilitating course, but shows also a disposition to undergo natural cure when the patient's resistance can be assisted by his circumstances and environment. The disease was recognized centuries ago as the king's evil, and was cured then by the royal touch. To-day there is unanimity of opinion that many varieties of treatment may achieve success, but that certainty of this is assured by none. The authors, therefore, while recognizing that different methods may be employed with equally excellent results by different workers, wish to set out a method of treatment based on the pathology of this disease that has achieved a fair measure of success in a reasonable amount of time.

The review deals with 119 consecutive cases of tuberculous cervical adenitis treated in Black Notley Sanatorium (Essex County Council) over a period of six years. The patients have been drawn from town and country alike, and both sexes and all ages have been accepted. Some had already received treatment by operation or other means before admission, but the majority were new and untreated cases. In general the treatment has consisted of a combination of constitutional and operative measures ; in the last four years there has been less delay in submitting patients to operation where such was indicated, but constitutional treatment has been the fundamental factor, and in no case was surgical extirpation of the tuberculous glands adopted as a primary measure.

\section{Sex Age Groups}

It will be seen from the table given below that seventy out of the 119 cases fall into the 6 to 15 years decade, and that the vast majority occur in childhood and adolescence, before the lymphatic system undergoes its natural decline.

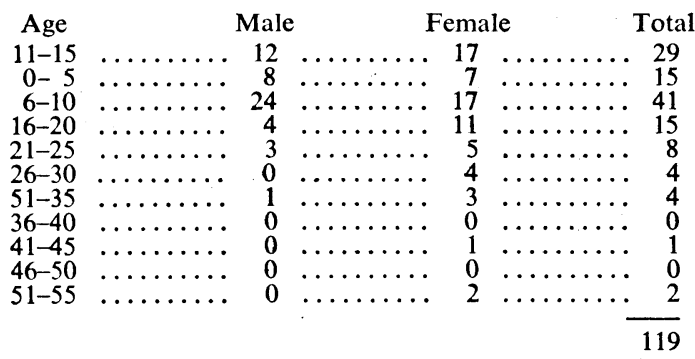

\section{Family History}

In this series of 119 cases there was no family history of tuberculosis in seventy-one, in twenty-five cases no note was made but there is good reason to believe it was negative, and in the remaining twenty-three cases the following was elicited: there was a history of phthisis in the family in nineteen cases, of glands in the neck in two cases, of tuberculosis of the knee in one case, and of tuberculous meningitis in one case. Thus in this series only 16 per cent. of patients had a near relative suffering from phthisis. This fact bears out the observation that the human form of bacillus is relatively uncommon in tuberculous glands. Many investigators have estimated the frequency of bovine tuberculosis in this disease affecting the cervical glands, and the following is a summary of their findings: Scotland, 90 per cent.; England, 65 per cent. ; U.S.A., 54 per cent. ; Germany, 25 per cent.

\section{Tonsillar Sepsis}

The cervical lymphatic glands drain the tonsil and lymphatic tissue of the nasopharynx, the first tissue to come into contact with ingested tubercle bacilli. If the lymphatic tissue of the nasopharynx and tonsil is unhealthy, and if as a result the cervical lymphatic glands are also abnormal, it may be that the frontiers are left poorly guarded against invasion of tuberculosis, which will then become established in the cervical glands. Howarth and Gloyne (Lancet, 1923, 1, 1202) found that the tubercle bacillus was present in the tonsil in about 5 per cent. of 300 cases of tonsillectomy in patients with cervical adenitis. Howarth stated that tuberculosis was a late and superadded infection upon a gland damaged as a result of focal sepsis in the drainage area.

Of the 119 cases in this series eighty-five had a history or signs of tonsillar disease, in twenty-one cases only the throat was normal, and in thirteen cases no note on this point was made. Of the eighty-five patients with abnormal tonsils thirty-one had been treated by tonsillectomy prior to admission, and twenty-two had their tonsils and adenoids removed during their stay in the sanatorium. In the remaining thirty-two cases, although the tonsils were not normal, for various reasons no tonsillectomy was performed. The findings in this series therefore tend to support the theory that disease in the lymphatic tissues of the nasopharynx is an important factor in the aetiology of tuberculous glands of the neck.

\section{Pathology}

Sources of tubercle bacilli other than the exhalations and sputum of consumptives and the milk from tuberculous cows seem to be of little importance, and from the frequency of bovine tuberculosis it may be concluded that milk is the great vehicle of infection. All evidence is in favour of the bacillus entering through the tonsil or surrounding lymphatic tissue and passing thence to the lymphatic barrier in the glands of the neck. As would be expected, the upper carotid chain is the group most often affected, although glands in the posterior triangle and the supraclavicular regions are not uncommonly involved, and it is not unusual to find evidence of disease in more than one group of glands in the neck. In only one case in this series was the cervical adenitis part of a generalized tuberculous lymphadenitis, this condition occurring typically in a negro child.

An interesting fact in this series is that in no case was the disease found to exist beyond the lymphatic system, and no case has shown secondary lesions such as pulmonary or bone tuberculosis. In tuberculosis of the lymphatic system there are evidences of great powers of resistance to the infection, and it may be that not only can this system withstand and overcome an infection which is not too heavy, but that such infection successfully protects the individual throughout life.

The tubercle bacilli, having established themselves in the affected gland, produce their characteristic lesions, and 
the gland undergoes a fleshy enlargement. If the infection is heavy or the resistance of the host poor the gland will undergo caseation in its central part and the surrounding parenchymatous tissues will be found infiltrated with oedema. This periadenitis is probably due to the more dilute toxins of the bacillus that have spread out from the main centre of infection, the caseous area. Neighbouring glands in the chain are invaded, and soon many of these swollen glands will be found matted together by periadenitis. The caseous pus may rupture the gland capsule and burrow to the surface, penetrating the deep fascia of the neck and becoming subcutaneous. A collar-stud abscess is then formed. The skin over the swelling is thus fixed either by the periadenitis or by the abscess, which if left alone will burst. This devitalized skin can at any time act as a path for secondary infecting organisms, and should such an infection occur either through the skin or by way of a sinus the cold abscess becomes an acute infective abscess.

In favourable cases of tuberculous lymphadenitis the gland does not proceed beyond the stage of fleshy enlargement, and with or without treatment shrinks to normal. It should be mentioned, however, that there is a rarer type of case in which many glands are enlarged in a way that superficially resembles Hodgkin's disease. These glands are multiple, discrete, mobile, and do not caseate. They do not respond well to constitutional treatment, and they recur after operative removal. An example of this type has been quoted below.

At any stage of the disease resolution may start as the natural resistance of the patient becomes effective. Periadenitis, the signal of an active and progressive infection by the tubercle bacillus, first disappears and the glands become more discrete, mobile, and firm to the touch. The caseous pus, if it has not been discharged, dries up and in time is calcified. The remaining small, hard, calcified gland may feed a sinus through which the whole gland can be extruded as a foreign body, thus completing the natural cure.

The above conception of the pathology of tuberculous lymphadenitis is the basis of the following classification and is the guide to treatment. The diseased glands are divided into three groups:

Group 1. Enlarged Glands with Periadenitis.-The glands may or may not contain pus or caseous material, and the periadenitis exists with or without an abscess or sinus. The periadenitis is due to tuberculous infection in an active stage, and there may be superadded a septic infection.

Group 2. Enlarged Glands without Periadenitis.These are cases in which either the infection has not been virulent or the periadenitis has subsided naturally or as a result of treatment. These glands may or may not have broken down.

Group 3. Cases of Residual Infection Following Natural or Artificial Attempts at Cure.-The glands in this group are usually small and hard, and may lie at the bottom of the sinus. Residual glands of this nature are found when complete resolution has not followed conservative treatment and in cases after operative removal without constitutional treatment.

The following table shows the cases in this series in the groups in which they were placed on admission:

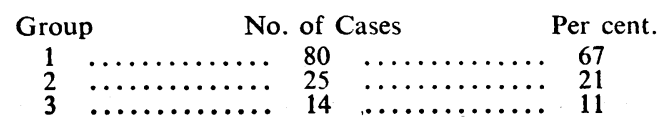

\section{Principles of Treatment}

The basis of the classification into three groups is increasing quiescence; the aim of treatment is to carry the case from Group 1 through Group 2 into the soundly healed state.

The Group 1 cases are treated by constitutional measures and minor operative procedures, and by this means the periadenitis will be abolished and tissue breakdown will cease. The glands may then be considered to have reached the stage of those included in Group 2. If reasonably rapid progress is being maintained under constitutional treatment by Group 2 cases this form of treatment should be continued. If there is lack of progress or if the patient's circumstances demand his return to work in the least possible time, radical operative removal of glands in this stage is to be performed. It will thus be seen that it is the more persistent and difficult cases which require radical operation. If the case is in Group 3, with a residual gland, operative removal is usually indicated; a persistent sinus which is not caused by a residual gland usually heals by constitutional measures. Finally operation may be employed for cosmetic reasons when the disease itself is quiescent.

\section{Details of Treatment}

Tuberculous lymphadenitis may be regarded as a local tuberculous lesion in a patient whose resistance to the disease is insufficient. Constitutional treatment, therefore, is of paramount importance to prevent progress or recrudescence of the disease. The chief factors in constitutional treatment are rest, open air, a liberal balanced diet with adequate vitamin supply, and heliotherapy.

With regard to rest, the patient should be restrained from all activity, but in our experience rest in bed for the whole day is not necessary unless the disease is very active. Fixation of the head and neck in sandbags or plaster has only rarely been used in this series. Natural sunlight and artificial light treatment have been found to be of value, both generally to improve the patient's resistance to tuberculosis and locally to ulcers of the neck following the breaking down of tuberculous glands. One of us (M. C. W.) has seen cases of tuberculous lymphadenitis treated by Dr. T. Gordon Pugh at Carshalton with radium applications used as an adjuvant to constitutional measures with good results. Of the use of $x$ rays in treatment we have no experience.

Reference has been made to the importance of tonsillar and pharyngeal sepsis in the pathology of tuberculous glands of the neck. The practice in cases showing signs of tonsillar disease has therefore been to perform tonsillectomy as soon as the patient is acclimatized and the general condition is sufficiently good to render the operation a matter of incident only. Following this treatment there is improvement in the general condition of the patient and in the rate of subsidence of the glands. It should be noted that the presence of retropharyngeal glands may complicate tonsillectomy, and in such cases we prefer to wait until a considerable amount of resolution has occurred in the gland so placed before advising the operation.

The treatment of a tuberculous abscess arising from a gland depends on whether superadded secondary infection has occurred or not. If there is secondary infection the pus should be evacuated by incision, and free drainage should be established. If there is no secondary infection an attempt should be made to treat the abscess by aspiration. It should be remembered, however, that as a 
general rule the success of aspiration in the treatment of a tuberculous abscess depends upon the employment of a technique in which the needle is passed into the abscess cavity through and across muscle. The needle track is thus closed by the approximation of the muscle fibres. We are indebted to Sir Henry Gauvain for instruction in this technique. In the neck this technique can rarely be used, as the abscesses are superficial and the needle cannot be inserted across the muscle. Furthermore, the pus in these cervical glandular abscesses is often caseous and does not aspirate readily through a needle, or, when it does, the aspirated cavity is small and refills so quickly that aspirations have to be repeated very often, and in spite of them the abscess frequently breaks down. The authors therefore reserve aspiration for all other forms of tuberculous abscess; but when it appears to be unlikely to succeed in the treatment of a tuberculous glandular abscess they empty the abscess through a small incision by means of gentle curettage and then re-suture the wound. The risk of secondary infection following this procedure is small, possibly because the tuberculous granulations which form a good nidus for organisms of secondary infections have been removed. The wound does not often heal by first intention, but the operation is followed by subsidence of periadenitis and in the size of the gland mass, and subsequent radical operation if performed is considerably facilitated.

There is a type of tuberculous skin involvement due to the breaking down of a tuberculous gland beneath the skin. An ulcer is formed the base of which consists of a deep layer of tuberculous granulations; these may be treated by "brass paste," as recommended by Sir Henry Gauvain. This paste causes sloughing of the granulations, leaving the ulcer bare, clean, and healthy. Alternatively, curettage of the granulations may be performed.

\section{Radical Operative Treatment}

The operation for radical excision of tuberculous glands of the neck is a controversial field in surgery. There is no doubt that it has produced many unsatisfactory results, due chiefly to the sequelae of recurrence, persistent sinus, muscle palsy, and ugly scars. Some of the cases in this series had been previously operated upon by radical excision with bad results. The causes of this failure can be almost wholly attributed to ill-advised operation before general treatment had converted the case from Group 1 stage to a stage of less activity. If operations are attempted in the presence of.periadenitis it will be found that the difficulties are very great, larger incisions are required, and diseased glands may be missed and nerves injured. The main contraindication for the operation, therefore, is active disease with periadenitis.

\section{Anaesthesia}

The anaesthetic must be one which can be administered without obstructing the surgeon. A basal anaesthetic with gas and oxygen is suitable, therefore, but at times it causes considerable venous congestion of the neck. Open ether, general contraindications being absent, is on the whole the most satisfactory anaesthetic, as there is less congestion and administration is simple and not complicated by bulky apparatus. It has already been noted that pulmonary lesions are rare when there is tuberculous disease in the neck glands.

\section{Operation}

The neck is turned to the sound side and an anaesthetic screen erected. A natural transverse crease in the neck over the tumour is chosen and a transverse collar incision is made, including any sinus or diseased skin that may be present. If the mass is extensive it is more satisfactory from the cosmetic point of view to make two or more transverse incisions, rather than a vertical one. The vertical incision, however restricted, produces a poor cosmetic result, and full exposure can be given by the transverse incision. The cut divides the platysma, the flaps are then dissected upwards and downwards, and a self-retaining retractor is inserted. If a sinus leading to the gland is visible, dissection of the gland is simplified by passing a director into the sinus and pulling up the gland. In any case the sterno-mastoid muscle is dissected backwards off the mass and partly divided if necessary. The mass of glands is then pulled backwards, and the fascial tissue on its anterior margin picked up and dissected forwards close to the gland until a line of cleavage is found. Gauze dissection will then sweep this fascia off the glands, and vessels will be identified and tied. As the gland is pulled upwards and backwards and dissection continued the internal jugular vein will be seen: This can be peeled off the glands, never being firmly adherent. The mass is then pulled down, and further dissection with scissors and gauze close to the gland reveals the posterior belly of the digastric muscle. The mass is cleared from this and gauze is packed into the depth of the wound. Next the sterno-mastoid is retracted and cleared off the mass; here, high up, the spinal accessory nerve will be seen entering the deep surface of the muscle. In order to clean and preserve it, division of the mass may be necessary. The gauze plug is now removed and in the depth of the wound the jugular vein and its anterior tributaries will be seen with the digastric muscle above. The gland mass is next dissected upwards towards the skull until the uppermost gland is reached. Above this the pedicle is clamped and the mass removed. It is essential that all glands which are enlarged and firm or caseous should be removed. Soft seedling glands may be left, and dissection need not be carried far afield to clear the chain, provided that no hard white glands are left. If in any part of the operation the gland mass is found to be ill defined and to involve important structures injury of which would be prejudicial to the patient, it is best to avoid risk of injury by curetting the affected part, as small residual areas of caseous tissue are not likely to prevent healing by first intention or to cause recurrence of disease if sufficient constitutional treatment has been given. When haemostasis has been secured the deep space is closed as far as possible by fine deep sutures, the platysma is united with the same material, and the skin closed with ophthalmic salmon-gut. A drain inserted for twenty-four hours in the posterior part of the wound is useful in preventing a haematoma, which may organize if any residual space is left. Stitches are loosely tied and are removed in four days; if this is done and the platysma properly sutured the cosmetic results should be excellent.

\section{Analysis of Immediate Results}

Before examining the tabled results below certain facts should be borne in mind.

1. The cases are grouped according to their state on admission.

2. Under the heading of conservative or constitutional treatment are included all surgical measures except formal excision.

3. Formal excision was required for the more intractable cases, therefore the duration of treatment is not a 
standard for comparison between constitutional and operative methods. Some cases would probably never have been completely cured by constitutional measures alone.

4. The results on discharge-that is, the immediate results-were judged on clinical grounds, taking into consideration the general health and the state of the gland areas and the overlying skin. "Good result " means that no glands were palpable, that the sinus or scar was soundly healed, and that the general health was normal.

5. Of the 119 cases admitted to the sanatorium, and considered above, only 109 are available for inclusion in the table of results, as for various reasons ten patients did not complete their treatment.

TABLE I.-Results of Treatment (109 cases)

\begin{tabular}{|c|c|c|c|c|c|c|}
\hline & \multicolumn{3}{|c|}{ Conservative } & \multicolumn{3}{|c|}{ Operative } \\
\hline & $\underset{1}{\text { Group }}$ & $\underset{2}{\text { Group }}$ & $\underset{3}{\text { Group }}$ & $\underset{1}{\text { Group }}$ & $\underset{2}{\text { Group }}$ & $\underset{3}{\text { Group }}$ \\
\hline $\begin{array}{l}\text { Average total length of stay } \\
\text { in sanatorium in months } \\
\text { Average length of pre- } \\
\text { operative sanatorium } \\
\text { treatment in months ... } \\
\text { Average length of post- } \\
\text { operative sanatorium } \\
\text { treatment in months } \\
\text { Good clinical results on } \\
\text { discharge. } \\
\text { Fair clinical results on dis- } \\
\text { charge } \\
\text { Poor clinical results on dis- } \\
\text { charge .. .. }\end{array}$ & $\begin{array}{r}24 \\
2 \\
3\end{array}$ & 3.25 & 3.0 & $\begin{array}{l}4.3 \\
2.9 \\
1.4 \\
45\end{array}$ & $\begin{array}{l}3.0 \\
2.0 \\
1.0 \\
16\end{array}$ & $\begin{array}{c}4.0 \\
3.0 \\
1.0 \\
6\end{array}$ \\
\hline No. of Cases .. & 29 & 6 & 7 & 45 & 16 & 6 \\
\hline
\end{tabular}

From the above table the following points may be noted concerning the cases in each group.

\section{GROUP 1 CASES ON ADMISSION}

The cases treated by conservative means required on an average 6.7 months to complete a clinical cure, and twenty-four out of twenty-nine gave a good result on discharge. The operative cases were under general treatment for an average period of 2.9 months before they passed from this group to Group 2 or 3 , and were then ready for operation. After operation one month of treatment was given to allow satisfactory healing of the wound. All the cases treated by operation gave good results.

\section{GROUP 2 CASES ON ADMISSION}

The six cases under constitutional treatment gave satisfactory results and were retained in the sanatorium for an average period of 3.25 months. The sixteen cases treated by operation all gave good results and were kept under constitutional treatment for an average period of two months before operation. During this time minor operative measures, such as aspiration, incision, and curettage or tonsillectomy, may have been performed, and the gland may have resolved to a Group 3 stage. A month was allowed after operation for the healing of the wound.

GROUP 3 CASES ON ADMISSION

The statistics in this group give no useful information, as the group contains such a variety of cases of residual infection that no generalization can be made about them. Thus there were cases with residual glands of long standing needing only a few weeks' treatment before operation, and cases with a residual sinus following an operation in a general hospital which had so far done well but which required a period of convalescent treatment to heal the sinus and to raise the resistance with a view to prevention of possible recurrence.

With regard to immediate results following treatment in all groups, it will be seen that they were good except in five cases of Group 1. There is no doubt, in view of our present experience, that the treatment of these five cases should have been completed by operation. It is noteworthy also that in this series of cases healing after excision occurred by first intention in every case but one.

\section{Analysis of Late Results}

The late results in this series of cases have been investigated with a view to ascertaining whether the principles of treatment outlined above have been successful in practice. The authors were fortunate in being able to procure the co-operation of the tuberculosis officers of the Essex County Council, to whom a questionary regarding the present condition of the patients was addressed. Information concerning eighty-one cases in the series was received. There is good reason for supposing that many of the cases about which information was not obtained did well, as some were taken off the register and others were given very good prognosis when they were discharged from the sanatorium. The following table embodies the information received in answer to the questionary:

TABLE II.-Summary of the Late Results of Treatment in the Series

\begin{tabular}{|c|c|c|c|c|c|c|c|}
\hline & & & & \multicolumn{2}{|c|}{$\begin{array}{l}\text { Constitutional } \\
\text { Treatment with } \\
\text { Formal Excision } \\
\quad \text { (54 Cases) }\end{array}$} & \multicolumn{2}{|c|}{$\begin{array}{c}\text { Constitutional } \\
\text { Treatment } \\
\text { only } \\
\text { (27 Cases) }\end{array}$} \\
\hline & & & & No. & Per cent. & No. & Per cent. \\
\hline \multicolumn{4}{|c|}{ Present condition of neck : } & & & & \\
\hline Satisfactory $\quad$.. & .. & .. & .. & 52 & 96 & 24 & 89 \\
\hline Unsatisfactory .. & .. & .. & .. & 2 & 4 & 3 & 11 \\
\hline Recurrence of glands & .. & .. & .. & 4 & 7 & 4 & 15 \\
\hline Further treatment & .. & .. & .. & 2 & 4 & 4 & 15 \\
\hline \multicolumn{8}{|l|}{ Cosmetic result : } \\
\hline Satisfactory $\quad .$. & .. & .. & .. & 49 & 90 & 23 & 85 \\
\hline Unsatisfactory .. & .. & .. & .. & 5 & 10 & 4 & 15 \\
\hline \multicolumn{2}{|c|}{ Further tuberculous lesions } & .. & .. & 0 & - & 0 & - \\
\hline
\end{tabular}

It will be seen from the table that the results taken as a whole are satisfactory, especially in view of the fact that many of the patients in the original series have had their names removed from the registers as no longer suffering from tuberculosis. The combination of constitutional treatment with formal excision gives slightly better results all round in spite of the fact that these cases were more severe. In considering the question of recurrence of glands, certain of the excision cases required another operation within a short time of the first one. In reality these recurrent glands were missed at the first operation and were not recent developments, as they were mostly caseous and broken down. The removal of a large superficial mass allows such glands to present more superficially in the neck. The reoperation cases were operated upon before the patients left the sanatorium, and such recurrences have not been included in the table. In a few cases the recurrence of glands mentioned in the table has not been sufficient to warrant further treatment.

If any note was made in the questionary that keloid scarring was present the cosmetic result was regarded as 
unsatisfactory. An interesting feature in late results is that no fresh foci of tuberculosis had been found to develop in any case. This supports the view that the lymphatic tissues of the body form a barrier to invasion by the tubercle bacillus, and when invasion is met with in this site blood-stream infections do not occur. This is also compatible with the fact that traces of old cervical adenitis are rare in cases under treatment in the sanatorium for tuberculosis at other sites.

\section{Causes of Failure}

Among the causes of failure must be included the type of gland already mentioned which becomes palpable owing to mechanical reasons, perhaps many months after a more superficial mass has been removed. True recurrence of the disease, however, is indicated only by the fresh development of tuberculosis in a gland previously unaffected. In this series cases of failure have been found to be due most commonly to incomplete treatment. Either the constitutional treatment was inadequate or excision was not carried out when it should have been. In at least one case tonsillectomy was omitted and the infected tonsil was the cause of failure. The following cases are quoted to illustrate the various causes of failure.

\section{INSUFFICIENT CONSTITUTIONAL TREATMENT}

Case $A$; a Man aged 30.-This patient was admitted with a breaking-down gland in the right posterior triangle; there was much periadenitis. After some weeks the periadenitis had subsided; at operation the gland mass was incompletely excised as there was some spread by abscess formation which was not clearly defined. The operation was finished by curettage. The wound healed by first intention in fourteen days. As the patient was anxious to return to business he was then discharged. One year later he developed a fresh tuberculous gland in the right submaxillary region. There was no trace of disease at the site of the previous lesion.

Case B; a Boy aged 6.-Admitted from a hospital where inadequate tonsillectomy had been performed. There was a mass of tuberculous glands behind the left sterno-mastoid. These were removed, and after four months' treatment in all the patient was discharged. He was readmitted a year later with an enlarged left submaxillary gland showing calcification. The tonsil on the left side was very unhealthy. During his second admission to the sanatorium tonsillectomy was performed and the left submaxillary gland removed. The causes of failure in this case were inadequate constitutional treatment in the first instance and failure of the original tonsillectomy.

Case $C ; a$ Boy aged 6.-Admitted suffering from tuberculous cervical adenitis with periadenitis and ulceration. After three months' conservative treatment this had subsided considerably, and it became necessary to transfer the child owing to pressure of beds. The parents refused this and took the child home. Recurrences subsequently occurred, requiring further treatment. This case should perhaps not be included in the series as treatment was not completed in the first instance.

\section{CASES IN WHICH CONSTITUTIONAL TREATMENT FAILED; OPERATION SHOULD HAVE BEEN PERFORMED TO COMPLETE CURE}

Case $A ; a$ Boy aged 5.-When admitted the patient hac a large mass of left submaxillary glands with periadenitis and ulceration. Ten months' constitutional treatment was given, and he was discharged with a persistent gland, which broke down again later. This was a "failed constitutional treatment" case which should have been treated by surgery at the Group 2 stage.

Case B; a Girl aged 12.--Admitted with breaking-down gland in the left side of 'he neck. After six months' con- stitutional treatment she was discharged with a small, hard gland which lay at the bottom of a healed sinus. Afterwards the sinus reopened: this would not have occurred if the gland which had reached the Group 3 stage had been removed before discharge.

\section{CASE IN WHich tONSILlECTOMY SHOULD HAVE BEEN} PERFORMED

A Girl aged 12.-When this patient was admitted she had a small mass of glands in the posterior triangle, which healed well after seven months' constitutional treatment. 'The tonsils were unhealthy, but were not removed. Four years after discharge there was recurrence at a fresh site in the submaxillary region. The patient was readmitted and tonsillectomy performed, with excision of glands.

\section{CASES IN WHICH CAUSE OF RECURRENCE IS NOT KNOWN}

Case A: a Boy aged 14.--Admitted with multiple discrete glands in both sides of neck, the size of marbles. $\mathrm{He}$ had had five previous operations, and the diagnosis of tuberculosis had been proved by pathological examination. He was treated by constitutional measures, including a plaster collar, for five months, and excision of glands on both sides of the neck was performed. He was then sent to a special school in the country. On his return home, however, recurrence started in the glands. These were of the non-caseating type.

Case B; a Woman of 25.-This patient had had three operations for tuberculous cervical glands, followed by constitutional treatment at the sanatorium on each occasion. A recent report states that there is a fresh recurrence of tuberculous glands.

\section{Conclusions}

In our opinion the conclusions below sủmmarize our experience of tuberculous glands of the neck derived from the collection and study of the cases forming this series.

1. The nasopharyngeal lymphatic tissue is the probabie path of entry for the tubercle bacillus in this disease.

2. Tuberculosis of the cervical lymph glands is a local dišease which does not give rise to metastatic lesions.

3. Treatment is basically by constitutional methods aided at the proper time by active surgical procedures.

4. When treatment is carried out according to the above plan results are satisfactory.

Our thanks are due to Sir Henry Gauvain, under whose supervision the work has been done; and to Dr. W. A. Bullough and the tuberculosis officers of the Essex County Council, for their information concerning the present condition of the patients in the series.

An interesting case of lead poisoning is described in the annual report on the health of the city of Sheffield, the cause of the poisoning being contaminated beer. It was found that the patient had been a frequent customer at an establishment where beer was conveyed from barrel to beer pump through lead pipes. Of samples of the beer which had remained in these pipes overnight one specimen showed a content of lead of one-third of a grain per gallon of beer. Immediate action by the health department resulted in the replacement of the lead pipes by others of suitable material. Other breweries and licensed clubs in the city were notified of the danger and undertakings to discontinue the use of lead pipes within a reasonable time were given. The mortality rate from diphtheria during the year was the highest since 1915. A record number of cases $(1,951)$ were admitted to the city fever hospital. In the majority of the severe cases the "gravis" type of $C$. diphtheriae continued to be isolated. It was found that these cases tended to become toxic at a very early stage. Of eightyfour deaths thirty-seven occurred in cases which had received antitoxin within the first three days of the illness. 